\title{
Hybrid task scheduling algorithm for high availability in cloud
}

\author{
Snehaa.K, Sivasankari.S \\ Asst. Professor, Dept of Information Technology, SRM University, Chennai, India
}

\begin{abstract}
Today, wherever we go we will find one thing or the other that is running in cloud. It has become an essential component in our everyday life. Many cloud service providers have been extensively working on improving the performance of the service provided. Highavailability in cloud is a place where various cloud service providers are extensively researching. High availability in cloud can vary depending on the scenario it is considered under. In our case we are going to see this in the form of response time for the request to get processed and how we can improve it. We are proposing an algorithm which will ensure the above and also provide an efficient model for analysing the same. We will also be configuring Reinforcement machine learning algorithm for training the load balancer and the server to process the request faster in a short duration of time.
\end{abstract}

Keywords: Load balancing; High Availablity; Vm scheduling;task scheduling;cloud computing.

\section{Intoduction}

Cloud computing is an very forward and burgoning technology,this permits data acess convienently for software and web based IT services.the major fuctionality of this is that the user only has topay for the servicer that he is using for the exact amount of time he is using, this ensures that the performance is high and very efficient compared to other technologies. The major technology which is also an important used here is virtualization which isolates the physiacl infrastructure with their required harware resources which are virtualised.It uses a vareity of processrs for system and alsoevaluvates a large volume of data of varoius applications.

The assets of Cloud are dynamic, various, selfautomatic and complicated in nature, making the management of those assets a perplex process. useful resource handling and load balancing are the two crucial problems of project scheduling in cloud computing [1]. The hassle of assignment scheduling on multiprocessor device is NP-complete. consequently, many researchers have proposed numerous heuristic or metaheuristic algorithms to address this trouble. those heuristics algorithms are in addition categorized into clustering algorithms, scheduling algorithms,etc. [2].

\section{A. High Availablity Architecture}

Network Load Balancing[3]:-Load balancing is a method to allocate obligations throughout two or extra computer systems, community, links, CPUs, tough force or different assets. Load balancing is used to dispensing a larger processing load to smaller processing nodes for boosting the overall performance of the machine. In the emerging technology surroundings it exceptionally requires load balancing to segregate the workload dynamically among all of the nodes present. network load balancing (usually referred to as twin-WAN routing or multihoming) is the potential to balance traffic throughout WAN hyperlinks without the use of complex routing protocols like BGP. It enhances the availability and scalability of net server packages inclusive of the ones used on web, FTP, firewall, proxy, virtual private network (VPN), and other mission-rucial servers. The particular and completely disbursed structure of network Load Balancing enables it to supply very high overall performance and failover safety, specifically in assessment with dispatcher-based Load balancers.

Server Clustering [4]:-Clustering linking two or greater servers to work collectively as a single system to deal with variable workloads or to provide endured operation inside the event one fails has been round because the earliest computing days. As the amount of information flowing through a employer's systems will increase, clustering will become greater essential because one server can't cope with the growing load. Clustering was specialised, requiring almost same computers and unique working gadget versions to live synchronized, as well as unique networking adapters to transport facts at excessive speeds among computers. As the recognition of cloud computing has grown,[5] however, clustering within the cloud has grow to be a truth. you can virtualize your clustering efforts and, with a 3rd-party cloud provider, get the ones resources off your IT branch's plate completely.

\section{Related Work}

In this phase, we describe the related work adequate challenge scheduling in cloud computing environment.

In paper [7] author proposed an approach for task scheduling algorithm primarily based on load balancing in cloud computing. This paper defined degree task scheduling based totally on the load balancing. This form of task scheduling can't most effective meet client's requirement but moreover provide excessive useful resource usage. This paper provided the implementation of an efficient and high-quality of provider (QoS) based totally MetaScheduler and Backfill technique based totally slight weight digital tool Scheduler for dispatching jobs.

In paper[8] author is going thru all the numerous task scheduling algorithms that may be used within the cloud generation. He additionally proposes an algorithms which can be used for 
measuring which of the parameters. This paper presented more than vital records about the algorithms and their operating development.

In paper [9] a brand new VM Load Balancing set of policies is Weighted active monitoring Load Balancing set of guidelines the of CloudSim equipment, for the Datacentre to efficiently load stability requests most of the available virtual machines assigning a weight, if you want to gain better typical overall performance parameters. proper right here VMs of diverse processing powers and the obligations/requests are assigned or allocated to the maximum powerful VM and then to the bottom and so forth.

\section{Proposed Framework}

Resource allocation and task scheduling are the major functionalities that degrade the performance of any system or technology. There has been may researches going on and many algorithms have been proposed to tackle the resource allocation and task scheduling problem in cloud computing. The three major steps to any task scheduling Process[10] is

-Discovering of Resources and filtering them in the datacentre brokers. It also collects all the related and important network information present.

- The target task or resource is selected from a range of resources based on the requirements and certain parametersof the task.

-The final step is that the required job is submitted to the concerned request.

The Best approach will be to define a model in which we will implement our algorithm this will prove to be not only thorough but also efficacious. We will be dividing our system into three major parts

-Load Balancing

-Server

-Data Recovery and backup

We will be focusing our research on the load balancing and the server part of the architecture. The will be configured with Network Load Balancing And Server Clustering will also be there This will help in increasing the performance of the high availability in cloud.

We will be comparing three major algorithms for our system a) Round Robin b)Ant colony Optimisation c)HTA Algorithm. This will make sure that the results produced by our algorithm are marginally better than the other two algorithms.

\section{1) Round Robin(RR)}

RoundRobin [9](RR) algorithm specializes inside the equity. RR makes use of the ring as its queue to shop jobs. each procedure in a queue has the equal execution time and it will likely be finished in turn. If a technique can't be completed sooner or later of its turn, it'll in all likelihood be stored decrease lower back to the queue watching for the subsequent turn. The advantage of RR algorithm is that each task can be finished in turn and that they don't should be waited for the previous one to get finished. however if the load is observed to be heavy, RR will take a long term to finish all of the jobs. The Cloud Sim toolkit helps RR scheduling approach for inner scheduling of jobs. The downside of RR is that the bigges job takes sufficient time for completion. The round Robin set of rules become designed based totally on the distribution of CPU time some of the scheduled tasks. at the equal context, all of the duties get on a queue listing whereas each undertaking get a small unit of CPU time (Quantum, commonly 10-100 milliseconds)[11]

\section{2) Ant Colony Optimisation(ATO)}

Ant colony optimization [12](ACO) is the only strategies used for solving the NP-entire troubles for example, touring salesman trouble, graph colouring hassle, vehicle routing hassle, scheduling problem, and so on. [12]. ACO receives its inspirations from the natural behaviour of ant colonies for looking meals and connecting with one any other via pheromone trails that are left at the back of with the aid of ants on the trails they travel. ACO approach is used for scheduling impartial tasks on the set of to be had resources with the intention to optimize makespan along with load balancing. To solve more than one targets concurrently by means of ant colony optimization they have got used the non-dominance type technique to address health capabilities, particularly, makespan and load balancing.

\section{3)HTA-LB}

Hybrid Task Scheduling-Load Balancing algorithm proposes to have the characteristics of Both the algorithms (ie) RR and ACO thereby having an efficacious result that is expected. we will be measuring the response time and the throughput of the jobs by performing the HTA-LB algorithm on these jobs.

\section{Experimental Results}

We will be using cloudsim package in java for doing our simulation and obtaining the results. Here we will configure the datacentre based on certain characteristics that are needed for doing our experiment.

Table 1: Input Parameters

\begin{tabular}{|l|l|}
\hline \multicolumn{2}{|c|}{ Table 1: Input Parameters } \\
\hline PARAMETERS & VALUE \\
\hline Datacenter & 2(DC1 and DC2) \\
\hline Dirtual Machine & $50(\mathrm{DC} 1)$ and 50(DC2) \\
\hline Number of Process MachineWise & Xen \\
\hline MIPS & 20 \\
\hline Data Centre VM Policy & 1000 \\
\hline Data Centre OS & Time Shared \\
\hline
\end{tabular}

\section{A) Response Time}

The response time is calculated nased on the task job arrival, delay and exedution time and hence the formula will be

$$
\mathrm{R}_{\mathrm{t}}=\mathrm{F}_{\mathrm{t}}+\mathrm{A}_{\mathrm{t}}-\mathrm{D}_{\mathrm{t}}
$$

As we can see by comparing the three major algorithms in the cloudsim tool, we found that the response time of our system is comparatively less than the other two algorithms.

Table 2: Experimental Results for different Algorithm

\begin{tabular}{|l|l|}
\hline \multicolumn{1}{|c|}{ Algorithm } & \multicolumn{1}{c|}{ Response Time(ms) } \\
\hline Round Robin & 244.51 \\
\hline Ant Colony Optimisation & 238.61 \\
\hline HTA-LB & 231.5 \\
\hline
\end{tabular}

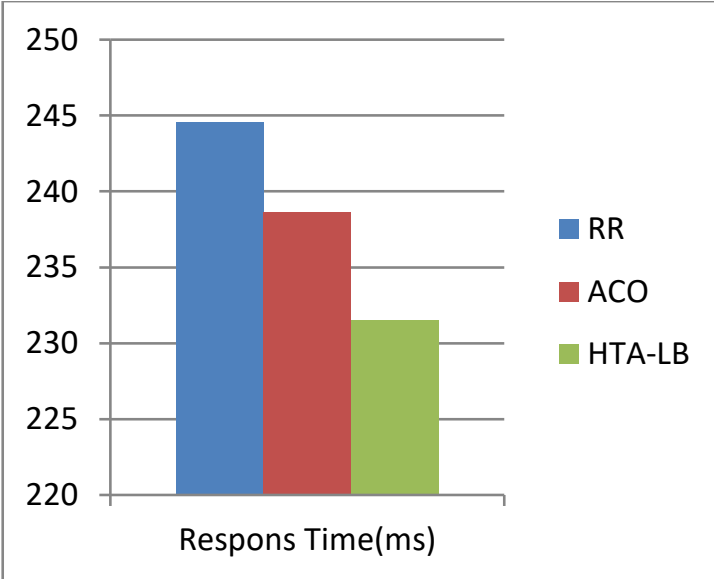

Fig. 1: Response Time Graph 


\section{Conclusion and Future Work}

The HTA-LB is used to proportion the weight to the available VM correctly. It improves the reaction time and minimizes the put off time. Subsequently the weight balancing is achieved. Numerous performance metrics which include availability, usage, and responsiveness used to analyse the effect of various strategies on each company and person point of views. In market-oriented vicinity, including the cloud computing, a particular evaluation of these parameters is required to quantify the presented QoS and opportunely control SLAs. This paper identifies that HTA-LB grid venture scheduling algorithm outperforms the round Robin and similarly spread algorithms in a heterogeneous dispensed surroundings. The experimental effects shows that response time is minimized as compared to round Robin and similar algorithms .This look at may be similarly prolonged with the aid of presenting a brand new hybrid algorithms to symbolize PaaS and SaaS cloud systems and to combine the mechanisms to seize VM migration and information middle consolidation elements for energy saving rules.

\section{References}

[1] Rathore, Neeraj, and Inderveer Chana. "Load balancing and job migration techniques in grid: a survey of recent trends." Wireless personal communications 79, no. 3 (2014): pp 2089-2125.

[2] Bala, Anju, and Inderveer Chana. "A survey of various workflow scheduling algorithms in cloud environment." In 2nd National Conference on Information and Communication Technology (NCICT),pp. 26-30. sn, 2011.

[3] Burya R Raman, R. Calheiros, R.N.(2009) "Modeling and Simulation of Scalable Cloud Environment and the Cloud Sim Toolkit: Challenges and Opportunities', IEEE publication 2009,pp1-11

[4] "Network Load Balancer "https://technet.microsoft.com/en-us/ library/cc725691(v=ws.11).aspx

[5] "Server Clustering "https://wordframe.com/docs/wiki/servercluster-definition

[6] Zou S. (2012) Analysis and Algorithm of Load Balancing Strategy of the Web Server Cluster System. In: Zhao M., Sha J. (eds)Communications and Information Processing. Communications in Computer and Information Science, vol 289. Springer, Berlin, Heidelberg.

[7] Dr.SudhaSadhasivam, R. Jayarani, Dr. N. Nagaveni, R. Vasanth Ram "Design and Implementation of an efficient Twolevel Scheduler for Cloud Computing Environment" In Proceedings of International Conference on Advances in Recent Technologies in Communication and Computing, 2009

[8] G. Guo-Ning and H. Ting-Lei, "Genetic Simulated Annealing Algorithm for Task Scheduling based on Cloud Computing Environment," In Proceedings of International Conference on Intelligent Computing and Integrated Systems, 2010, pp. 60-63

[9] Jasmin James, Dr.BhupendraVerma "Efficient Vm Load BalancinAlgorithim For A Cloud Computing Environment " In Proceeding of International Journal on Computer Science and Engineering (IJCSE) Vol. 4 No. 09, Sep 2012

[10] Dr. Amit Agarwal, Saloni Jain "Efficient Optimal Algorithm of Task Scheduling in Cloud Computing Environment" In International Journal of Computer Trends and Technology (IJCTT) - volume 9 number 7- Mar 2014

[11] G. T. Hicham, EL.Chaker, Cloud Computing CPU Allocation and Scheduling Algorithms using CloudSim Simulator International Journal of Electrical and Computer Engineering, Vol 6, No 4, 2016

[12] Dorigo, M., Gambardella, L.M.: Ant colony system: a cooperative learning approach to the traveling salesman problem. IEEE Trans. Evol. Comput. 1(1), pp53-66 (1997).

[13] S.V.Manikanthan and V.Rama"Optimal Performance of Key Predistribution Protocol In Wireless Sensor Networks" International Innovative Research Journal of Engineering and Technology ,ISSN NO: 2456-1983,Vol-2,Issue -Special -March 2017.

[14] T. Padmapriya and V.Saminadan, "Handoff Decision for Multiuser Multiclass Traffic in MIMO-LTE-A Networks", 2nd
International Conference on Intelligent Computing, Communication \& Convergence (ICCC-2016) - Elsevier PROCEDIA OF COMPUTER SCIENCE, vol. 92, pp: 410-417, August 2016. 